\title{
Corner singularities of Maxwell interface and eddy current problems
}

\author{
Martin Costabel, Monique Dauge, and Serge Nicaise
}

\begin{abstract}
The aim of this work is to provide a description of the corner asymptotics for the solutions of Maxwell equations in and outside a conductor body and to investigate the limit as the ratio permittivity/conductivity tends to zero (the eddy current limit). Corner singularities of the Maxwell transmission problem and also of the eddy current model have been described elsewhere $[6,7]$. Here we concentrate on the uniform behavior with respect to the small parameter describing the eddy current limit - analyticity of the singular functions and stability of the decomposition of the fields into regular and singular parts.
\end{abstract}

\section{Introduction}

We consider the time-harmonic Maxwell equations in a medium with a high conductivity in one part and isolating in the other part. We are not interested in scattering aspects here, but will study the local regularity of the fields, in particular near corners of the conductor. Since the questions are local, we can assume from the outset that the domain is bounded. Let therefore $\Omega \subset \mathbb{R}^{3}$ be a bounded domain decomposed into the two subdomains $\Omega_{C}$, the conductor, and $\Omega_{E}$, which corresponds to the exterior isolator. For the sake of simplicity, we assume that $B:=\partial \Omega_{C}$ is connected, and that $\partial \Omega_{E}=\partial \Omega \cup B$. The conductivity $\sigma$ is a constant $\sigma_{C}>0$ in $\Omega_{C}$ and vanishes in $\Omega_{E}$. The electric permittivity $\varepsilon$ and the magnetic permeability $\mu$ are supposed to be positive constants on $\Omega_{C}$ and on $\Omega_{E}$. The frequency $\omega$ is a fixed positive constant. The eddy current model describes the situation where the quotient $\omega \varepsilon / \sigma_{C}$ is very small.

We consider the case where $B$ has a conical singularity. As is well known, in the neighborhood of this corner point the electrical field will, in general, be unbounded. All possible singularities for the solutions of the time-harmonic Maxwell equations near conical corner points have been described in [5] for the case of perfect conductor boundary conditions, in [6] for interface conditions, and in [7] for

Received by the editors April 24, 2003.

1991 Mathematics Subject Classification. Primary 35J; Secondary 00A00.

Key words and phrases. Maxwell, Eddy current, Corners, Singularities. 
the eddy current model. In all these cases, it has been shown that the singular functions can be obtained from corresponding scalar problems for the Laplace equation. Therefore the analytical tools for singularity analysis, mainly Kondrat'ev's Mellin transform based technique, are applied to the well-studied boundary value and interface problems for the Laplacian.

If, however, we want to describe the behavior of the singularities as the full Maxwell problem tends towards the eddy current problem, we need to use tools that have been developed for the situation of singularity problems depending on a parameter $([4,14,16])$. Since the coefficients of the operator are complex, we cannot expect any simplification, but have to take into account all the possible complications that may appear in such situations, such as "crossing" and "branching" of the exponents. In order to get a stable description of the singular behavior, we no longer can reduce everything to the Laplace operator. Instead we have to use Mellin transformation directly for the Maxwell system. The corresponding constructions of spaces and operators do not seem to exist in the literature.

In the eddy current limit, the PDE problem itself changes its type from a pure transmission problem for a strongly elliptic second order system to a mixture of one-sided boundary conditions and transmission conditions for a system of Maxwell type in the conductor and of Laplace type with a divergence-free constraint in the isolator. On the other hand, from the point of view of the description of singularities, the eddy current limit is a regular perturbation problem.

Thus it is not hard to show that in this limit, not only the solution of the Maxwell problem converges in the energy norm to the solution of the eddy current problem, but also the singularity exponents converge at the same time. This has been proved in [7].

The continuity of the solution and of the singularity exponents does not imply, however, that in any decomposition of the solution into regular and singular parts, all the terms - regular part, singular functions, and coefficients of the singular functions - will also depend continuously on the small parameter $\delta$ that characterizes the eddy current limit. This is the problem we are studying here.

In general, when the singularity exponent in the eddy current limit is of multiplicity higher than one, a "naïve" decomposition into a regular part and individual singular functions will produce coefficients tending to infinity as $\delta \rightarrow$ 0 . We show how to choose bases of singular functions that lead to coefficients continuous as $\delta \rightarrow 0$, and we prove stability for the decomposition into regular and singular parts in this case.

For the Mellin analysis, the "Mellin symbol", i. e. the angular part of the Maxwell transmission problem, has to be considered in a strong form, that is, on a level of regularity above the level of the energy norm related to the variational formulation. This means in particular that the natural transmission conditions now appear explicitly in the formulation of the Mellin symbol. We indicate in section 5 how they are obtained. We also show how the classification of the singularities into types 1,2 , and 3 is obtained from the Mellin analysis. 


\section{Maxwell and eddy current problems}

Let $\omega>0$ be a fixed frequency. The time harmonic Maxwell equations are

$$
\left\{\begin{aligned}
\operatorname{curl} \mathbf{E} & =-i \omega \mu \mathbf{H} & & \text { in } \Omega, \\
\operatorname{curl} \mathbf{H} & =(i \omega \varepsilon+\sigma) \mathbf{E}+\mathbf{j}_{0} & & \text { in } \Omega, \\
\mathbf{E} \times \mathbf{n} & =0 \text { and } \mathbf{H} \cdot \mathbf{n}=0 & & \text { on } \partial \Omega,
\end{aligned}\right.
$$

$\mathbf{E}$ (resp. $\mathbf{H}$ ) is the electric (resp. magnetic) field and $\mathbf{j}_{0}$ is the source current density which is supposed to be a $L^{2}(\Omega)$ field with support in $\Omega_{C}$ and to be divergence free, i.e. $\operatorname{div} \mathbf{j}_{0}=0$ in $\Omega$. Note that the assumption on $\operatorname{div} \mathbf{j}_{0}$ is equivalent to

$$
\operatorname{div} \mathbf{j}_{0}=0 \text { in } \Omega_{C} \quad \text { and } \quad \mathbf{j}_{0} \cdot \mathbf{n}=0 \text { on } B .
$$

Thus, taking the divergence of the second equation of (1.1), we obtain the following equation on the divergence of $\mathbf{E}$ :

$$
\operatorname{div}(i \omega \varepsilon+\sigma) \mathbf{E}=0 \quad \text { in } \quad \Omega .
$$

The time-harmonic eddy current problem $[2,3,1,9]$ reads

$$
\left\{\begin{aligned}
\operatorname{curl} \mathbf{E} & =-i \omega \mu \mathbf{H} & & \text { in } \Omega, \\
\operatorname{curl} \mathbf{H} & =\sigma \mathbf{E}+\mathbf{j}_{0} & & \text { in } \Omega . \\
\mathbf{E} \times \mathbf{n} & =0 \text { and } \mathbf{H} \cdot \mathbf{n}=0 & & \text { on } \partial \Omega,
\end{aligned}\right.
$$

Let us write $\mathbf{E}_{C}=\left.\mathbf{E}\right|_{\Omega_{C}}$ and $\mathbf{E}_{E}=\left.\mathbf{E}\right|_{\Omega_{E}}$.

Taking the divergence of the second equation of (1.3), instead of condition (1.2) we only obtain $\operatorname{div} \mathbf{E}_{C}=0$ in $\Omega_{C}$ and $\mathbf{E}_{C} \cdot \mathbf{n}=0$ on $B$. These conditions have to be completed by the gauge conditions:

$$
\operatorname{div} \mathbf{E}_{E}=0 \text { in } \Omega_{E} \text { and } \int_{B} \mathbf{E}_{E} \cdot \mathbf{n} \mathrm{d} S=0 .
$$

\subsection{Eddy current limit}

Following [3, Ch.4], we consider the eddy current limit as the limiting situation when the quantities $\omega \varepsilon_{C} / \sigma_{C}$ and $\omega \varepsilon_{E} / \sigma_{C}$ are small. For a conducting material, the permittivity $\varepsilon_{C}$ is of the same order of magnitude as $\varepsilon_{E}$ (also denoted $\varepsilon_{0}$ ), but $\varepsilon_{C} / \sigma_{C}$ is very small. For moderate frequencies $\omega$ the quantities $\omega \varepsilon_{C} / \sigma_{C}$ and $\omega \varepsilon_{E} / \sigma_{C}$ are still small. Let us fix two numbers $\hat{\varepsilon}_{C}$ and $\hat{\varepsilon}_{E}$ which are of the same order as $\sigma_{C}$ and such that there exists $\delta>0$ (thus $\delta$ is small)

$$
\varepsilon_{C}=\delta \hat{\varepsilon}_{C} \quad \text { and } \quad \varepsilon_{E}=\delta \hat{\varepsilon}_{E} .
$$

Thus we can write (defining by the same token the complex electric transmission coefficient $\alpha$ )

$$
i \omega \alpha:=i \omega \varepsilon+\sigma= \begin{cases}i \omega \delta \hat{\varepsilon}_{C}+\sigma_{C} & \text { in } \Omega_{C} \\ i \omega \delta \hat{\varepsilon}_{E} & \text { in } \Omega_{E} .\end{cases}
$$

We fix $\sigma_{C}, \omega, \hat{\varepsilon}_{C}$ and $\hat{\varepsilon}_{E}$. The eddy current limit is the limit $\delta \rightarrow 0$. 


\subsection{Strong electric formulations}

We use the electric approach consisting in eliminating $\mathbf{H}$ from equations (1.1)(1.2) and (1.3)-(1.4). We denote by $\mathbf{E}^{\delta}$ for $\delta>0$ according to (1.6), the solution of equations (1.1)-(1.2) and by $\mathbf{E}^{0}$ the solution of (1.3)-(1.4). We note that we can write the equations satisfied by $\mathbf{E}^{\delta}$ in a unified way for $\delta>0$ and $\delta=0$ :

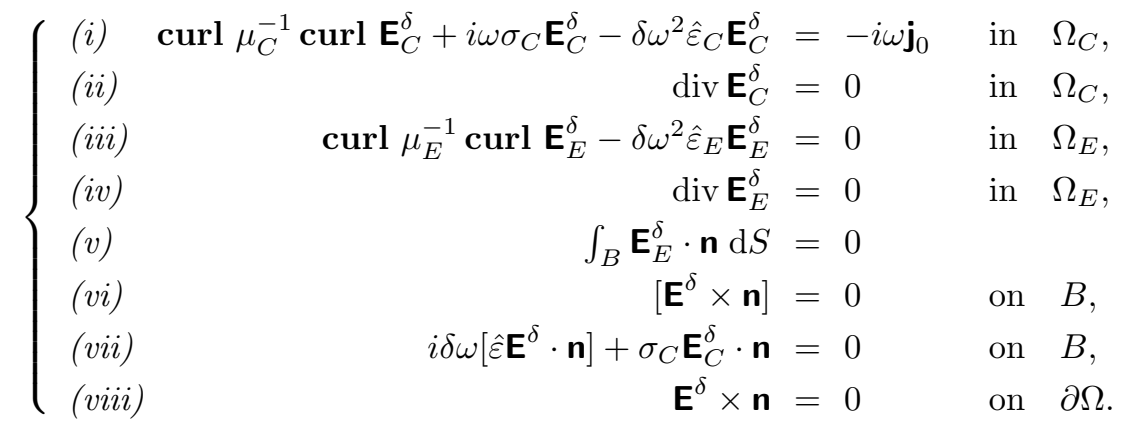

\subsection{Variational formulations}

The variational space which we will use is independent of $\delta$, i.e. suitable for both the Maxwell and the eddy current problem. Let $H_{0}(\mathbf{c u r l}, \Omega)$ be the standard space

$$
H_{0}(\operatorname{curl}, \Omega)=\left\{\mathbf{u} \in L^{2}(\Omega)^{3}: \operatorname{curl} \mathbf{u} \in L^{2}(\Omega)^{3}, \quad \mathbf{u} \times \mathbf{n}=0 \text { on } \partial \Omega\right\} .
$$

Our variational space $\mathbf{Y}(\Omega)$ is defined as

$$
\mathbf{Y}(\Omega)=\left\{\mathbf{u} \in H_{0}(\mathbf{c u r l}, \Omega): \operatorname{div} \mathbf{u}_{C} \in L^{2}\left(\Omega_{C}\right), \operatorname{div} \mathbf{u}_{E} \in L^{2}\left(\Omega_{E}\right), \int_{B} \mathbf{E} \cdot \mathbf{n}=0\right\}
$$

equipped with the norm

$$
\|\mathbf{u}\|_{\mathbf{Y}(\Omega)}^{2}=\|\mathbf{u}\|_{0, \Omega}^{2}+\|\mathbf{c u r l} \mathbf{u}\|_{0, \Omega}^{2}+\left\|\operatorname{div} \mathbf{u}_{C}\right\|_{0, \Omega_{C}}^{2}+\left\|\operatorname{div} \mathbf{u}_{E}\right\|_{0, \Omega_{E}}^{2} .
$$

There is a full family of sesquilinear forms $a^{\delta}$ on $\mathbf{Y}(\Omega)$ adapted to a regularized variational formulation of the problem (1.7) for $\delta \geq 0$ : We arbitrarily fix some positive parameter $\gamma$ (possibly different in $\Omega_{C}$ and $\Omega_{E}$ ) and we define $a^{\delta}$ as follows: For $\mathbf{u}, \mathbf{v} \in \mathbf{Y}(\Omega)$ :

$$
\begin{aligned}
a^{\delta}(\mathbf{u}, \mathbf{v})= & \int_{\Omega}\left(\mu^{-1} \operatorname{curl} \mathbf{u} \cdot \operatorname{curl} \overline{\mathbf{v}}-\delta \omega^{2} \hat{\varepsilon} \mathbf{u} \cdot \overline{\mathbf{v}}\right) \mathrm{d} x+i \omega \int_{\Omega_{C}} \sigma_{C} \mathbf{u} \cdot \overline{\mathbf{v}} \mathrm{d} x \\
& +\int_{\Omega_{C}} \gamma_{C} \operatorname{div} \mathbf{u}_{C} \operatorname{div} \overline{\mathbf{v}}_{C} \mathrm{~d} x+\int_{\Omega_{E}} \gamma_{E} \operatorname{div} \mathbf{u}_{E} \operatorname{div} \overline{\mathbf{v}}_{E} \mathrm{~d} x .
\end{aligned}
$$

Lemma 1.1. Let the positive constants $\mu_{C}, \mu_{E}, \hat{\varepsilon}_{C}, \hat{\varepsilon}_{E}, \sigma_{C}$ and $\omega$ be fixed. Then there exists $\delta_{0}>0$ such that for all $\delta \in\left[0, \delta_{0}\right]$, a $a^{\delta}$ is strongly coercive on $\mathbf{Y}(\Omega)$ : $\exists c_{0}>0, \forall \delta \in\left[0, \delta_{0}\right], \forall \mathbf{u} \in \mathbf{Y}(\Omega)$

$$
\operatorname{Re}\left((1-i) a^{\delta}(\mathbf{u}, \mathbf{u})\right) \geq c_{0}\|\mathbf{u}\|_{\mathbf{Y}(\Omega)}^{2} .
$$


For all $\delta \in\left[0, \delta_{0}\right]$, we consider the variational problem:

$$
\text { Find } \mathbf{E} \in \mathbf{Y}(\Omega) \text { s. t. } a^{\delta}(\mathbf{E}, \mathbf{v})=-i \omega\left(\mathbf{j}_{0}, \mathbf{v}\right)_{\Omega_{C}}, \quad \forall \mathbf{v} \in \mathbf{Y}(\Omega),
$$

where $(\cdot, \cdot)_{D}$ is the $L^{2}(D)^{3}$ hermitian inner product.

The following result is proved in [7]:

Theorem 1.2. Under the assumptions of Lemma 1.1, there holds for all $\delta \in\left[0, \delta_{0}\right]$ :

(i) There exists a unique solution $\mathbf{E}^{\delta}$ to problem (1.8).

(ii) The solution $\mathbf{E}^{\delta}$ satisfies all equations in (1.7).

(iii) The norms of the $\mathbf{E}^{\delta}$ in $\mathbf{Y}(\Omega)$ are uniformly bounded:

$$
\exists C>0, \quad \forall \delta \in\left[0, \delta_{0}\right], \quad\left\|\mathbf{E}^{\delta}\right\|_{\mathbf{Y}(\Omega)} \leq C .
$$

(iv) As $\delta \rightarrow 0, \mathbf{E}^{\delta} \rightarrow \mathbf{E}^{0}$ and we have the convergence estimate

$$
\exists C>0, \quad \forall \delta \in\left[0, \delta_{0}\right], \quad\left\|\mathbf{E}^{\delta}-\mathbf{E}^{0}\right\|_{\mathbf{Y}(\Omega)} \leq C \delta .
$$

\section{Localization at a conical point}

Let us assume that $\Omega_{C}$ has a smooth boundary except at one point, say $O$, where it coincides with the tip of a cone $\Gamma_{C}$. The solid angle $G_{C}:=\Gamma_{C} \cap \mathbb{S}^{2}$ is a smooth domain in $\mathbb{S}^{2}$. In a neighborhood of $O$, the exterior domain $\Omega_{E}$ coincides with the open cone $\Gamma_{E}$ such that $\bar{\Gamma}_{C} \cup \bar{\Gamma}_{E}=\mathbb{R}^{3}$ and $\Gamma_{C} \cap \Gamma_{E}=\emptyset$. Let $G_{E}:=\Gamma_{E} \cap \mathbb{S}^{2}$. Let $(\rho, \vartheta)$ be spherical coordinates centered in $O$.

As far as elliptic boundary value or transmission problems on domains with conical points are concerned, the standard tool for the investigation of the structure of their solution is the Mellin transform defined for all $\mathbf{u} \in \mathcal{C}_{0}^{\infty}\left(\mathbb{R}^{3} \backslash\{0\}\right)$ and all $\lambda \in \mathbb{C}$ by

$$
\mathbf{u}=\mathbf{u}(\rho, \vartheta) \quad \longmapsto \quad \mathfrak{M} \mathbf{u}(\lambda)=\mathbf{U}(\lambda, \vartheta):=\int_{0}^{\infty} \rho^{-\lambda} \mathbf{u}(r, \vartheta) \frac{\mathrm{d} \rho}{\rho} .
$$

The argument in $[5,6,7]$ is that the equivalent regularized variational formulation (1.8) provides an equivalent elliptic transmission problem where the standard Kontrat'ev approach [11] applies. This is the reason why in these works only the structure of singularities is investigated.

If we want to investigate the possibility of a stable asymptotics with respect to the parameter $\delta$, we have to revisit the Mellin approach, in order to look for a Mellin symbol with meromorphic inverse which should depend analytically on $\delta$ (and acting between a couple of spaces independent from $\delta$ ).

\subsection{Ellipticity}

We integrate by parts in the variational problem (1.8) and find (with $\mathbf{u}=\mathbf{E}^{\delta}$ )

$$
\begin{cases}\text { (i) } \operatorname{curl} \mu_{C}^{-1} \operatorname{curl} \mathbf{u}_{C}-\nabla \gamma_{C} \operatorname{div} \mathbf{u}_{C}-\omega^{2} \alpha_{C} \mathbf{u}_{C}=-i \omega \mathbf{j}_{0} & \text { in } \Omega_{C}, \\ \text { (ii) } \operatorname{curl} \mu_{E}^{-1} \operatorname{curl} \mathbf{u}_{E}-\nabla \gamma_{E} \operatorname{div} \mathbf{u}_{E}-\omega^{2} \alpha_{E} \mathbf{u}_{E}=0 & \text { in } \Omega_{E},\end{cases}
$$


with the essential boundary conditions, - we recall that $\alpha$ is defined in (1.6):

$$
\begin{cases}\text { (i) }[\mathbf{u} \times \mathbf{n}]=0 & \text { on } B \\ \text { (ii) }[\alpha \mathbf{u} \cdot \mathbf{n}]=0 & \text { on } B\end{cases}
$$

which we complement by the Neumann type transmission conditions

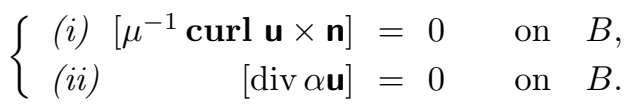

Proposition 2.1. Let $L_{C}$ and $L_{E}$ be the principal parts of the operators in (2.1). There exists $\delta_{0}>0$ such that for all $\delta \in\left[0, \delta_{0}\right]$ the set of transmission conditions (2.2) and $(2.3)$ covers $^{1}$ the couple of operators $\left(L_{C}, L_{E}\right)$ at any smooth point of the interface $B$.

Proof. Let us take $\delta=0$. We check that the $6 \times 6$ determinant obtained after partial Fourier transform at any point of the interface and reduction to the interface by the interior equations $L_{C} \mathbf{u}=0, L_{E} \mathbf{u}=0$ (symbol at the interface - cf standard covering boundary conditions) is non-zero for any real $\xi=\left(\xi_{1}, \xi_{2}\right) \neq 0$.

The corresponding determinant is therefore non-zero for $\delta$ small enough.

\subsection{Local regularity}

We deduce that our solution $\mathbf{u}$ corresponding to a charge density $\mathbf{j}_{0}$ in $L^{2}\left(\Omega_{C}\right)$ has an optimal local regularity up to the interface outside the corner $O$ : for any neighborhood $\mathcal{V}$ such that $O \notin \overline{\mathcal{V}}$ :

$$
\mathbf{u}_{C} \in H^{2}\left(\Omega_{C} \cap \mathcal{V}\right) \quad \text { and } \quad \mathbf{u}_{E} \in H^{2}\left(\Omega_{E} \cap \mathcal{V}\right)
$$

Let $\chi$ be a smooth cut-off function which is $\equiv 1$ in a neighborhood of $O$ and has its support in the region where $\Omega_{C}$ and $\Omega_{E}$ coincide with the cones $\Gamma_{C}$ and $\Gamma_{E}$ respectively. Still denoting $\chi \mathbf{u}$ by $\mathbf{u}$, we are left with the following problem, instead of (2.1)-(2.3):

$$
\begin{cases}\text { (i) } \operatorname{curl} \mu_{C}^{-1} \operatorname{curl} \mathbf{u}_{C}-\nabla \gamma_{C} \operatorname{div} \mathbf{u}_{C}\left(=L_{C} \mathbf{u}_{C}\right)=\mathbf{j}_{C} & \text { in } \Gamma_{C}, \\ \text { (ii) } \operatorname{curl} \mu_{E}^{-1} \operatorname{curl} \mathbf{u}_{E}-\nabla \gamma_{E} \operatorname{div} \mathbf{u}_{E}\left(=L_{E} \mathbf{u}_{E}\right)=\mathbf{j}_{E} & \text { in } \Gamma_{E},\end{cases}
$$

where $\mathbf{j}_{C}$ and $\mathbf{j}_{E}$ belong to $L^{2}\left(\Gamma_{C}\right)^{3}$ and $L^{2}\left(\Gamma_{E}\right)^{3}$ respectively (we have used the fact that $\mathbf{u}$ belongs to $L^{2}\left(\mathbb{R}^{3}\right)^{3}$ to put the term of order zero into the RHS),

$$
\begin{cases}\text { (i) }[\mathbf{u} \times \mathbf{n}]=0 & \text { on } I, \\ \text { (ii) }[\alpha \mathbf{u} \cdot \mathbf{n}]=0 & \text { on } I,\end{cases}
$$

where $I$ denotes the interface $\partial \Gamma_{C}=\partial \Gamma_{E}$, and

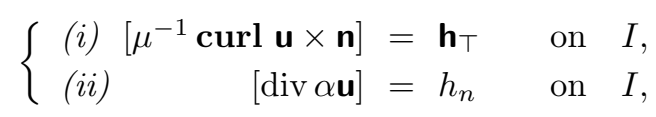

\footnotetext{
${ }^{1}$ In a sense extended from the classical Shapiro-Lopatinskii condition, [12, 15].
} 
where $\mathbf{h}_{\top} \in H^{1 / 2}(I)^{2}$ and $h_{n} \in H^{1 / 2}(I)$. Since the support of $\mathbf{h}_{\top}$ and $h_{n}$ is away from zero and infinity there also holds:

$$
\rho^{-1 / 2} \mathbf{h}_{\top} \in L^{2}(I)^{2} \quad \text { and } \quad \rho^{-1 / 2} h_{n} \in L^{2}(I) .
$$

A standard homogeneity argument based on the a priori estimate between the two nested annuli $\mathcal{A}_{j}=\left\{x: 2^{-j}<\rho<2^{j}\right\}, j=1,2$ :

$$
\begin{aligned}
\left\|\mathbf{u}_{C}\right\|_{H^{2}\left(\Gamma_{C} \cap \mathcal{A}_{1}\right)}+\left\|\mathbf{u}_{E}\right\|_{H^{2}\left(\Gamma_{E} \cap \mathcal{A}_{1}\right)} \leq C\left(\left\|\mathbf{j}_{C}\right\|_{L^{2}\left(\Gamma_{C} \cap \mathcal{A}_{2}\right)}+\left\|\mathbf{j}_{E}\right\|_{L^{2}\left(\Gamma_{E} \cap \mathcal{A}_{2}\right)}\right. \\
\left.+\left\|\mathbf{h}_{\top}\right\|_{H^{1 / 2}\left(I \cap \mathcal{A}_{2}\right)}+\left\|h_{n}\right\|_{H^{1 / 2}\left(I \cap \mathcal{A}_{2}\right)}\right)
\end{aligned}
$$

yields the weighted regularity for $\mathbf{u}_{C}$ and $\mathbf{u}_{E}$ :

$$
\rho^{|\alpha|} \partial^{\alpha} \mathbf{u}_{C} \in L^{2}\left(\Gamma_{C}\right) \quad \text { and } \quad \rho^{|\alpha|} \partial^{\alpha} \mathbf{u}_{E} \in L^{2}\left(\Gamma_{E}\right), \quad \forall \alpha,|\alpha| \leq 2 .
$$

\section{Mellin transform}

For each fixed $\delta \geq 0$, we can apply to problem (2.4)-(2.6) the standard tools of the Mellin transform and residue formula. Let us recall that the Mellin symbol of an operator $A$ homogeneous of degree $m$ with constant coefficients is $\mathbb{C} \ni \lambda \mapsto \mathfrak{A}(\lambda)$ where

$$
A\left(\partial_{x}\right)=\rho^{-m} \mathfrak{A}\left(\vartheta ; \rho \partial_{\rho}, \partial_{\vartheta}\right) \text { and } \mathfrak{A}(\lambda):=\mathfrak{A}\left(\vartheta ; \lambda, \partial_{\vartheta}\right) .
$$

If $\mathbf{U}(\lambda)$ is the Mellin transform of $\mathbf{u}$, then the Mellin transform $\mathbf{F}(\lambda)$ of $\rho^{m} A \mathbf{u}$ is $\mathfrak{A}(\lambda) \mathbf{U}(\lambda)$. Practically, we have the relation

$$
A\left(\partial_{x}\right)\left(\rho^{\lambda} \mathbf{U}(\vartheta)\right)=\rho^{\lambda-m} \mathfrak{A}(\lambda) \mathbf{U}(\lambda) .
$$

\subsection{Mellin symbol}

In the case of our problem, we define a Mellin symbol acting between spaces which do not depend on $\lambda$ of course, but also not on $\delta$. We take as source space

$$
\mathbf{Y}^{+}\left(G_{C}, G_{E}\right)=\left\{\mathbf{U} \in L^{2}\left(\mathbb{S}^{2}\right): \mathbf{U}_{C} \in H^{2}\left(G_{C}\right), \mathbf{U}_{E} \in H^{2}\left(G_{E}\right),[\mathbf{U} \times \mathbf{n}]=0\right\}
$$

and as target space

$$
\mathbf{Z}\left(G_{C}, G_{E}\right)=L^{2}\left(G_{C}\right)^{3} \times L^{2}\left(G_{E}\right)^{3} \times H^{3 / 2}(J) \times H^{1 / 2}(J)^{2} \times H^{1 / 2}(J),
$$

where we recall that $G_{C}=\Gamma_{C} \cap \mathbb{S}^{2}$ and $G_{E}=\Gamma_{E} \cap \mathbb{S}^{2}$, and $J$ denotes $\partial G_{C} \cap \partial G_{E}$. Let us fix $\lambda \in \mathbb{C}$. Our Mellin symbol $\mathfrak{L}(\lambda)$ is defined as follows

$$
\begin{aligned}
\mathfrak{L}(\lambda): \quad \mathbf{Y}^{+}\left(G_{C}, G_{E}\right) & \longrightarrow \mathbf{Z}\left(G_{C}, G_{E}\right) \\
\left(\mathbf{U}_{C}, \mathbf{U}_{E}\right) & \longmapsto \mathbf{F}=\left(\mathbf{J}_{C}, \mathbf{J}_{E}, G, \mathbf{H}_{\top}, H_{n}\right),
\end{aligned}
$$

with

$$
\begin{gathered}
L_{C}\left(\partial_{x}\right)\left(\rho^{\lambda} \mathbf{U}_{C}(\vartheta)\right)=\rho^{\lambda-2} \mathbf{J}_{C}(\vartheta), \quad L_{E}\left(\partial_{x}\right)\left(\rho^{\lambda} \mathbf{U}_{E}(\vartheta)\right)=\rho^{\lambda-2} \mathbf{J}_{E}(\vartheta) \\
{\left[\alpha \rho^{\lambda} \mathbf{U}(\vartheta) \cdot \mathbf{n}\right]=\rho^{\lambda} G(\vartheta)} \\
{\left[\mu^{-1} \operatorname{curl} \rho^{\lambda} \mathbf{U}(\vartheta) \times \mathbf{n}\right]=\rho^{\lambda-1} \mathbf{H}_{\top}(\vartheta), \quad\left[\operatorname{div} \alpha \rho^{\lambda} \mathbf{U}(\vartheta)\right]=\rho^{\lambda-1} H_{n}(\vartheta) .}
\end{gathered}
$$

As a standard consequence of the ellipticity (Proposition 2.1), we obtain that

- $\mathfrak{L}(\lambda)$ is invertible except for $\lambda$ in a discrete set $\mathfrak{S} \subset \mathbb{C}$, 
- $\lambda \mapsto \mathfrak{L}(\lambda)^{-1}$ is meromorphic,

- at each pole $\lambda \in \mathfrak{S}$, the range of the polar part of $\mathfrak{L}^{-1}$ is finite dimensional,

- in each strip of the form $\xi_{1} \leq \operatorname{Re} \lambda \leq \xi_{2}$ there are at most a finite number of elements of $\mathfrak{S}$.

The elements $\lambda$ of $\mathfrak{S}$ are the poles of $\mathfrak{L}(\lambda)^{-1}$ and they are also called singular exponents because they are the possible degrees of homogeneity of the singular parts in the conical asymptotics of solutions.

\subsection{Splitting in regular and singular parts}

As a consequence of the regularity and support properties of the data, $c f(2.7)$, the Mellin transform $\lambda \mapsto \mathbf{F}(\lambda)$ of

$$
\left(\rho^{2} \mathbf{f}_{C}, \rho^{2} \mathbf{f}_{E}, 0, \rho \mathbf{h}_{\top}, \rho h_{n}\right)
$$

is holomorphic for $\lambda$ in the half-plane $\operatorname{Re} \lambda<\frac{1}{2}$ with values in the space $\mathbf{Z}\left(G_{C}, G_{E}\right)$, whereas, thanks to (2.8), the Mellin transform $\lambda \mapsto \mathbf{U}(\lambda)$ of $\mathbf{u}$ is holomorphic for $\lambda$ in the half-plane $\operatorname{Re} \lambda<-\frac{3}{2}$ with values in the space $\mathbf{Y}^{+}\left(G_{C}, G_{E}\right)$. Moreover, there holds

$$
\mathfrak{L}(\lambda) \mathbf{U}(\lambda)=\mathbf{F}(\lambda), \quad \forall \lambda, \operatorname{Re} \lambda \leq-\frac{3}{2}
$$

The function $\lambda \mapsto \mathfrak{L}(\lambda)^{-1} \mathbf{F}(\lambda)$ is a meromorphic extension of $\mathbf{U}(\lambda)$ to the strip $-\frac{3}{2} \leq \operatorname{Re} \lambda<\frac{1}{2}$. As standard in the KONDRAT'Ev Cauchy residue analysis we obtain

$$
\mathbf{u}=\mathbf{u}_{\mathrm{reg}}-\frac{1}{2 i \pi} \int_{\gamma} \rho^{\lambda} \mathfrak{L}(\lambda)^{-1} \mathbf{F}(\lambda) \mathrm{d} \lambda
$$

where $\gamma$ is a simple curve surrounding all the poles of $\mathfrak{L}(\lambda)^{-1}$ in the strip $-\frac{3}{2} \leq$ $\operatorname{Re} \lambda<\frac{1}{2}$, and $\mathbf{u}_{\text {reg }}$ is the regular part satisfying, if $\mathfrak{S} \cap\left\{\lambda: \operatorname{Re} \lambda=\frac{1}{2}\right\}$ is empty:

$$
\rho^{|\alpha|-2} \partial^{\alpha} \mathbf{u}_{\mathrm{reg}, C} \in L^{2}\left(\Gamma_{C}\right) \quad \text { and } \quad \rho^{|\alpha|-2} \partial^{\alpha} \mathbf{u}_{\mathrm{reg}, E} \in L^{2}\left(\Gamma_{E}\right), \quad \forall \alpha,|\alpha| \leq 2 .
$$

Note that, in particular, the regular part $\mathbf{u}_{\text {reg }}$ is $H^{2}$ in any neighborhood of zero inside $\Gamma_{C}$ and $\Gamma_{E}$.

The properties of the polar part of $\mathfrak{L}^{-1}$ inherited from the ellipticity imply that the residue in (3.3) (the singular part) spans a finite dimensional space.

\section{Stable asymptotics with respect to $\delta$}

We now trace the dependency with respect to $\delta$ as $\delta \rightarrow 0$ in the above decomposition (3.3). We fix the right hand side $\mathbf{j}_{0}$ and consider $\mathbf{u}^{\delta}:=\chi \mathbf{E}^{\delta}$ the localized solution of problem (1.8). Since the form $a^{\delta}$ depends analytically on $\delta$, the solution $\mathbf{E}^{\delta}$ also depends analytically on $\delta$ in $\mathbf{Y}(\Omega)$ for $\delta$ in a neighborhood of $\delta=0$. Therefore, the localized solutions $\mathbf{u}^{\delta}$ and the associated right-hand sides $\left(\mathbf{f}_{C}^{\delta}, \mathbf{f}_{E}^{\delta}, \mathbf{h}_{\mathrm{T}}^{\delta}, h_{n}^{\delta}\right)$ depend analytically on $\delta$. 
Thus, with $\lambda \mapsto \mathbf{F}^{\delta}(\lambda)$ the Mellin transform of

$$
\left(\rho^{2} \mathbf{f}_{C}^{\delta}, \rho^{2} \mathbf{f}_{E}^{\delta}, 0, \rho \mathbf{h}_{\top}^{\delta}, \rho h_{n}^{\delta}\right)
$$

we finally have the splitting of $\mathbf{u}^{\delta}$ for each $\delta$ according to (3.3):

$$
\mathbf{u}^{\delta}=\mathbf{u}_{\text {reg }}^{\delta}-\frac{1}{2 i \pi} \int_{\gamma} \rho^{\lambda} \mathfrak{L}^{\delta}(\lambda)^{-1} \mathbf{F}^{\delta}(\lambda) \mathrm{d} \lambda
$$

where $\mathfrak{L}^{\delta}$ is the Mellin symbol of problem (2.4)-(2.6) with $\alpha$ depending on $\delta$ via formula (1.6).

\subsection{Spaces of singularities and characteristic polynomials}

Definition 4.1. For a $\delta \geq 0$ and a contour $\gamma$ which does not meet any pole of $\mathfrak{L}^{\delta}(\lambda)^{-1}$, we denote by $\mathbf{S}_{\gamma}^{\delta}$ the finite dimensional space spanned by the residues

$$
\frac{1}{2 i \pi} \int_{\gamma} \rho^{\lambda} \mathfrak{L}^{\delta}(\lambda)^{-1} \mathbf{F}(\lambda) \mathrm{d} \lambda
$$

for all holomorphic $\lambda \mapsto \mathbf{F}(\lambda)$ with values in $\mathbf{Z}\left(G_{C}, G_{E}\right)$. We denote by $m_{\gamma}^{\delta}$ its dimension.

We have the classical result, see [13] for example:

Lemma 4.2. For $\delta$ and $\gamma$ as in the definition above, let $D$ be the interior region such that $\partial D=\gamma$. Then

$$
m_{\gamma}^{\delta}=\sum_{\nu \in D \cap \mathfrak{S}^{\delta}} m^{\delta}(\nu)
$$

where $\mathfrak{S}^{\delta}$ is the set of poles of $\mathfrak{L}^{\delta}(\lambda)^{-1}$ and $m^{\delta}(\nu)$ is the sum of the lengths of all Keldysh chains associated with the pole $\nu$.

Definition 4.3. We call characteristic polynomial of $\mathfrak{L}^{\delta}$ inside $\gamma$ :

$$
P_{\gamma}^{\delta}(\lambda)=\prod_{\nu \in D \cap \mathfrak{S}^{\delta}}(\lambda-\nu)^{m^{\delta}(\nu)}
$$

The operator valued function $\lambda \mapsto P_{\gamma}^{\delta}(\lambda) \mathfrak{L}^{\delta}(\lambda)^{-1}$ is holomorphic in $\bar{D}$.

From the "operator valued Rouché formula" of GoHBERG-Sigal [8], we find:

Lemma 4.4. The following representation holds for $P_{\gamma}^{\delta}(\mu)$ when $\mu \notin \bar{D}$ :

$$
P_{\gamma}^{\delta}(\mu)=\exp \left\{\operatorname{tr} \frac{1}{2 i \pi} \int_{\gamma} \mathfrak{L}^{\delta}(\lambda)^{\prime} \mathfrak{L}^{\delta}(\lambda)^{-1} \log (\mu-\lambda) d \lambda\right\} .
$$

Here we fix the contour $\gamma$ so that $\mathfrak{L}^{0}(\lambda)^{-1}$ has no pole on $\gamma$. Therefore, for $0 \leq \delta \leq \delta_{0}$ with $\delta_{0}>0$ small enough, $\mathfrak{L}^{\delta}(\lambda)^{-1}$ has still no poles on $\gamma$. The analytic dependency of $\mathfrak{L}^{\delta}(\lambda)^{-1}$ on $\delta$ when $\lambda \in \gamma$ implies that there holds:

Corollary 4.5. The coefficients of the characteristic polynomial $P_{\gamma}^{\delta}$ depend analytically on $\delta$ for $0 \leq \delta \leq \delta_{0}$. In particular its degree does not depend on $\delta$ for $0 \leq \delta \leq \delta_{0}$ : we denote it by $m_{\gamma}$. 


\subsection{Stable asymptotics: general result}

Let us recall the notion of "distance" between two subspaces $E$ and $F$ of the same Hilbert space $H, c f[10]$ :

$$
\operatorname{dist}(E, F)=\max _{\mathbf{u} \in E} \min _{\mathbf{v} \in F} \frac{\|\mathbf{u}-\mathbf{v}\|_{H}}{\|\mathbf{u}\|_{H}}
$$

In general this distance is not symmetric. However, if $E$ and $F$ are finite dimensional subspaces satisfying $\operatorname{dist}(E, F)<1$ and $\operatorname{dist}(F, E)<1$, then $\operatorname{dim} E=\operatorname{dim} F$ and $\operatorname{dist}(E, F)=\operatorname{dist}(F, E)$. that

Again as a consequence of the analytic dependency of $\mathfrak{L}^{\delta}(\lambda)^{-1}$ on $\delta$ we find

and that there holds

$$
\operatorname{dist}\left(\mathbf{S}_{\gamma}^{0}, \mathbf{S}_{\gamma}^{\delta}\right)=\operatorname{dist}\left(\mathbf{S}_{\gamma}^{\delta}, \mathbf{S}_{\gamma}^{0}\right)=\mathcal{O}(\delta)
$$

Theorem 4.6. We can choose a basis $\mathbf{s}_{m}^{\delta}, m=1, \ldots, m_{\gamma}$, of $\mathbf{S}_{\gamma}^{\delta}$ depending analytically on $\delta$. The decomposition (4.1) of the localized solution $\mathbf{u}^{\delta}=\chi \mathbf{E}^{\delta}$ of problem (1.8) can be written

$$
\mathbf{u}^{\delta}=\mathbf{u}_{\mathrm{reg}}^{\delta}+\sum_{m=1}^{m_{\gamma}} c_{m}^{\delta} \mathbf{s}_{m}^{\delta}, \quad \text { with } \quad\left[0, \delta_{0}\right] \ni \delta \mapsto c_{m}^{\delta} \in \mathbb{C} \text { analytic } .
$$

with $\mathbf{u}_{\mathrm{reg}, C}^{\delta}$ in $H^{2}\left(\Omega_{C}\right)$ and $\mathbf{u}_{\mathrm{reg}, E}^{\delta}$ in $H^{2}\left(\Omega_{E}\right)$.

Let $\nu_{1}^{0}, \ldots, \nu_{d}^{0}$ be the distinct elements of $\mathfrak{S}^{0} \cap D$. Let $\gamma_{1}, \ldots, \gamma_{d}$ be contours around each of them and such that their interior regions $D_{1}, \ldots, D_{d}$ are pairwise disjoint. For $0 \leq \delta \leq \delta_{0}$ with $\delta_{0}>0$ small enough, $\gamma_{1}, \ldots, \gamma_{d}$ do not intersect $\mathfrak{S}^{\delta}$. We clearly have

$$
\mathbf{S}_{\gamma}^{\delta}=\bigoplus_{j=1}^{d} \mathbf{S}_{\gamma_{j}}^{\delta} .
$$

According to Corollary 4.5, each characteristic polynomial $P_{\gamma_{j}}^{\delta}$ has coefficients depending analytically on $\delta$. The roots of $P_{\gamma_{j}}^{\delta}$ form the cluster of singular exponents $\nu_{i}^{\delta}$ which tend to $\nu_{j}^{0}$ as $\delta \rightarrow 0$.

\subsection{Stable asymptotics: cases of degrees 1 and 2}

In particular, if the degree of $P_{\gamma_{j}}^{\delta}$ is one, the situation is "simple" as described in the following proposition.

Proposition 4.7. Let $\nu^{0}=\nu_{j}^{0} \in \mathfrak{S}^{0} \cap D$. If its multiplicity $m^{0}\left(\nu^{0}\right)$ is 1 , then there exists a unique element $\nu^{\delta}$ of $\mathfrak{S}^{\delta} \cap D$ which tends to $\nu^{0}$ as $\delta \rightarrow 0$. Moreover, $\delta \mapsto \nu^{\delta}$ is analytic and a basis $\mathbf{s}^{\delta}$ of $\mathbf{S}_{\gamma_{j}}^{\delta}$ can be chosen of the form

$$
\mathbf{s}^{\delta}(\rho, \vartheta)=\rho^{\nu^{\delta}} \mathbf{U}^{\delta}(\vartheta)
$$

where $\delta \mapsto \mathbf{U}^{\delta}$ is analytic in $\delta$ with values in $\mathbf{Y}^{+}\left(G_{C}, G_{E}\right)$. 
Although unpredictable in general, the simplicity of limiting exponents of singularity is generic. Nevertheless, the general question of stable asymptotics with respect to $\delta$ keeps its interest.

If the characteristic polynomial $P_{\gamma_{j}}^{\delta}$ is of degree 2 , the situation is more complicated, but still possible to describe:

Proposition 4.8. Let $\nu^{0}=\nu_{j}^{0} \in \mathfrak{S}^{0} \cap D$. If its multiplicity $m^{0}\left(\nu^{0}\right)$ is 2 , then there exists at least one and at most two elements $\nu_{i}^{\delta}, i=1,2$, of $\mathfrak{S}^{\delta} \cap D$ which tend to $\nu^{0}$ as $\delta \rightarrow 0$.

Let us assume that the pole of $\mathfrak{L}(\lambda)^{-1}$ in $\nu^{0}$ is of order 2 and that $\nu_{1}^{\delta} \neq \nu_{2}^{\delta}$ for all $\delta \neq 0$ small enough. Then we can choose a basis of $\mathbf{S}_{\gamma_{j}}^{0}$ of the form

$$
\mathbf{s}^{0}(\rho, \vartheta)=\rho^{\nu^{0}} \mathbf{U}^{0}(\vartheta) \quad \text { and } \quad \mathbf{t}^{0}(\rho, \vartheta)=\mathbf{s}^{0}(\rho, \vartheta) \log \rho+\rho^{\nu^{0}} \mathbf{V}^{0}(\vartheta) .
$$

Moreover, for all $\delta \neq 0$ small enough there exist two singular functions $\mathbf{s}_{i}^{\delta}, i=1,2$ of the form

$$
\mathbf{s}_{1}^{\delta}(\rho, \vartheta)=\rho^{\nu} \mathbf{U}_{1}^{\delta}(\vartheta) \quad \text { and } \quad \mathbf{s}_{2}^{\delta}(\rho, \vartheta)=\rho^{\nu_{2}^{\delta}} \mathbf{U}_{2}^{\delta}(\vartheta)
$$

such that

(i) $\mathbf{s}_{i}^{\delta}$ tends to $\mathbf{s}^{0}$ as $\delta \rightarrow 0, i=1,2$,

(ii) the two functions

$$
\frac{\mathbf{s}_{1}^{\delta}-\mathbf{s}_{2}^{\delta}}{\nu_{1}^{\delta}-\nu_{2}^{\delta}} \quad \text { and } \quad \mathbf{s}_{1}^{\delta}+\mathbf{s}_{2}^{\delta}
$$

are a basis of $\mathbf{S}_{\gamma_{j}}^{\delta}$ depending analytically on $\delta$. The first function tends to $\mathbf{t}^{0}$ and the second one to $2 \mathbf{s}^{0}$ as $\delta \rightarrow 0$.

Proof. The first assertions are classical.

Let us prove the existence of $\mathbf{s}_{i}^{\delta}$ satisfying the next assertions of the proposition. Let us denote for short the "small" contour surrounding $\nu^{0}$ by $\gamma$ instead of $\gamma_{j}$. By construction $P_{\gamma}^{0}(\lambda) \mathfrak{L}^{0}(\lambda)^{-1}$ is holomorphic in the region $\bar{D}$ surrounded by $\gamma$. As the pole $\nu^{0}$ is double, there exists $\mathbf{F}(\lambda)$ holomorphic in $\bar{D}$ such that $\left(\lambda-\nu^{0}\right) \mathfrak{L}^{0}(\lambda)^{-1} \mathbf{F}(\lambda)$ has a pole of order 1 in $\nu^{0}$. Let us define

$$
\mathbf{U}^{\delta}(\lambda):=P_{\gamma}^{\delta}(\lambda) \mathfrak{L}^{\delta}(\lambda)^{-1} \mathbf{F}(\lambda)
$$

Thus $\mathbf{U}^{\delta}\left(\nu^{0}\right)$ is not zero and the residue of $\rho^{\lambda}\left(\lambda-\nu^{0}\right) P_{\gamma}^{\delta}(\lambda)^{-1} \mathbf{U}^{\delta}(\lambda)$ is an element of $\mathbf{S}_{\gamma}^{0}$, which we denote by $\mathbf{s}^{0}$ :

$$
\mathbf{s}^{0}(\rho, \vartheta)=\rho^{\nu_{0}^{\delta}} \mathbf{U}^{\delta}\left(\nu_{0}^{\delta}\right) .
$$

The function $\lambda \mapsto \mathbf{U}^{\delta}(\lambda)$ is holomorphic in $\bar{D}$ and analytic in $\delta$. The residues of

are

$$
\lambda \longmapsto \rho^{\lambda} \frac{\mathbf{U}^{\delta}(\lambda)}{P_{\gamma}^{\delta}(\lambda)} \quad \text { in } \quad \nu_{1}^{\delta} \text { and } \nu_{2}^{\delta}
$$

$$
\left(\nu_{1}^{\delta}-\nu_{2}^{\delta}\right)^{-1} \rho^{\nu_{1}^{\delta}} \mathbf{U}^{\delta}\left(\nu_{1}^{\delta}\right) \quad \text { and } \quad\left(\nu_{2}^{\delta}-\nu_{1}^{\delta}\right)^{-1} \rho^{\nu_{2}^{\delta}} \mathbf{U}^{\delta}\left(\nu_{2}^{\delta}\right)
$$


respectively, and define elements of $\mathbf{S}_{\gamma}^{\delta}$. Let us set

$$
\mathbf{s}_{i}^{\delta}:=\rho^{\nu_{i}^{\delta}} \mathbf{U}^{\delta}\left(\nu_{i}^{\delta}\right), \quad i=1,2 .
$$

From (4.5), (4.6), the continuity of $\delta \mapsto \nu_{i}^{\delta}$ as $\delta \rightarrow 0$ and the regularity of $(\delta, \lambda) \mapsto$ $\mathbf{U}^{\delta}(\lambda)$, we deduce that $\mathbf{s}_{i}^{\delta}$ tend to $\mathbf{s}^{0}$ as $\delta \rightarrow 0$. We check that

$$
\frac{1}{2 i \pi} \int_{\gamma} \rho^{\lambda} P_{\gamma}^{\delta}(\lambda)^{-1} \mathbf{U}^{\delta}(\lambda) \mathrm{d} \lambda=\frac{\mathbf{s}_{1}^{\delta}-\mathbf{s}_{2}^{\delta}}{\nu_{1}^{\delta}-\nu_{2}^{\delta}}
$$

and

$$
\frac{1}{2 i \pi} \int_{\gamma} \rho^{\lambda} P_{\gamma}^{\delta}(\lambda)^{-1}\left(2 \lambda-\nu_{1}^{\delta}-\nu_{2}^{\delta}\right) \mathbf{U}^{\delta}(\lambda) \mathrm{d} \lambda=\mathbf{s}_{1}^{\delta}+\mathbf{s}_{2}^{\delta} .
$$

They both belong to $\mathbf{S}_{\gamma}^{\delta}$ and, since $\nu_{1}^{\delta}+\nu_{2}^{\delta}$ depends analytically on $\delta$, they both depend analytically on $\delta$ too.

General stable behaviors with respect to $\rho$ for more general characteristic polynomials are investigated in [4] and [16].

\section{Maxwell singularities}

In [5] it is proved that the singularities of the electric fields at corners and edges of a perfectly conducting polyhedral body all derive from scalar potentials, namely via three different types:

Type 1 are gradients of singularities of the Dirichlet Laplacian.

Type 2 are such that their curl is equal to the gradient of singularities of the Neumann Laplacian.

Type 3 are such that their divergence is a singularity of the Dirichlet Laplacian.

In $[6,7]$ we have simply applied the same procedure as in $[5]$ to describe the three different types in the case of transmission and eddy current problems. In this section, we are going to develop the arguments leading to such a description in these cases.

We investigate the structure of the elements of $\mathbf{S}_{\gamma}^{\delta}$. Let $\mathbf{s}^{\delta}$ belong to $\mathbf{S}_{\gamma}^{\delta}$ : There exits $\mathbf{F}$ holomorphic such that

$$
\mathbf{s}^{\delta}=\int_{\gamma} \rho^{\lambda} \mathfrak{L}^{\delta}(\lambda)^{-1} \mathbf{F}(\lambda) \mathrm{d} \lambda .
$$

Let $\mathfrak{L}^{\delta}=\mathfrak{L}^{\delta}\left(\rho \partial_{\rho}\right)$ be the collection of interior and interface operators

$$
\mathbf{u} \mapsto \mathfrak{L}^{\delta} \mathbf{u}=\left(\rho^{2} L_{C} \mathbf{u}_{C}, \rho^{2} L_{E} \mathbf{u}_{E},[\alpha \mathbf{u} \cdot \mathbf{n}], \rho\left[\mu^{-1} \operatorname{curl} \mathbf{u} \times \mathbf{n}\right], \rho[\operatorname{div} \alpha \mathbf{u}]\right),
$$

with $\alpha$ defined in (1.6), compare with (2.4)-(2.6). We have

$$
\mathfrak{L}^{\delta} \mathbf{s}^{\delta}=\int_{\gamma} \rho^{\lambda} \mathfrak{L}^{\delta}(\lambda) \mathfrak{L}^{\delta}(\lambda)^{-1} \mathbf{F}(\lambda) \mathrm{d} \lambda=\int_{\gamma} \rho^{\lambda} \mathbf{F}(\lambda) \mathrm{d} \lambda=0 .
$$

Let us fix $\delta \in\left[0, \delta_{0}\right]$, and thus $\alpha$ which is given by (1.6). From now on, we drop the exponent $\delta$. 
In other words, $\mathbf{s}$ is a solution of the elliptic interface problem (2.4)-(2.6) with zero right hand side:

$$
\begin{cases}\text { (i) } \operatorname{curl} \mu_{C}^{-1} \operatorname{curl} \mathbf{s}_{C}-\nabla \gamma_{C} \operatorname{div} \mathbf{s}_{C}=0 & \text { in } \Gamma_{C}, \\ \text { (ii) } \operatorname{curl} \mu_{E}^{-1} \operatorname{curl} \mathbf{s}_{E}-\nabla \gamma_{E} \operatorname{div} \mathbf{s}_{E}=0 & \text { in } \Gamma_{E},\end{cases}
$$

$$
\begin{aligned}
& \begin{cases}\text { (i) }[\mathbf{s} \times \mathbf{n}]=0 & \text { on } I, \\
\text { (ii) }[\alpha \mathbf{s} \cdot \mathbf{n}]=0 & \text { on } I,\end{cases}
\end{aligned}
$$

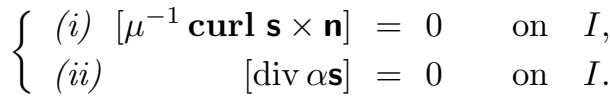

\subsection{Uncoupled systems}

The key of the investigation of solutions $\mathbf{s}$ of (5.1)-(5.3) is the introduction of the auxiliary unknowns

$$
q \text { s.t. }\left\{\begin{array} { l l } 
{ q _ { C } = \operatorname { d i v } \mathbf { s } _ { C } } & { \text { in } \Gamma _ { C } , } \\
{ q _ { E } = \operatorname { d i v } \mathbf { s } _ { E } } & { \text { in } \Gamma _ { E } , }
\end{array} \text { and } \boldsymbol { \psi } \text { s.t. } \left\{\begin{array}{l}
\boldsymbol{\psi}_{C}=\mu_{C}^{-1} \text { curl } \mathbf{s}_{C} \quad \text { in } \Gamma_{C}, \\
\boldsymbol{\psi}_{E}=\mu_{E}^{-1} \text { curl } \mathbf{s}_{E} \quad \text { in } \Gamma_{E} .
\end{array}\right.\right.
$$

Taking the divergence of equations (5.1), we find that $q_{C}$ and $q_{E}$ are harmonic. Condition (5.3) (ii) gives that $[\alpha q]=0$. Taking the normal component (with respect to the interface $I$ ) of equations (5.1), we find that

$$
\begin{aligned}
\gamma_{C} \partial_{n} \operatorname{div} \mathbf{s}_{C} & =\operatorname{curl}_{\top}\left(\mu_{C}^{-1} \operatorname{curl} \mathbf{s}_{C}\right)_{\top} \\
\gamma_{E} \partial_{n} \operatorname{div} \mathbf{s}_{E} & =\operatorname{curl}_{\top}\left(\mu_{E}^{-1} \operatorname{curl} \mathbf{s}_{E}\right)_{\top}
\end{aligned}
$$

Thanks to (5.3) (i), we obtain that the jump of $\operatorname{curl}_{\top}\left(\mu^{-1} \text { curl s) }\right)_{\top}$ is zero, whence $\left[\gamma \partial_{n} \operatorname{div} \mathbf{s}\right]=0$, i.e. $\left[\gamma \partial_{n} q\right]=0$. When $\delta \neq 0$, we can write equivalently that $\left[\alpha^{-1} \gamma \partial_{n}(\alpha q)\right]=0$. Summing up, we have obtained if $\delta \neq 0$

$$
\begin{cases}\Delta q_{C}=0 & \text { in } \Gamma_{C}, \\ \Delta q_{E}=0 & \text { in } \Gamma_{E}, \\ {[\alpha q]=0, \quad\left[\alpha^{-1} \gamma \partial_{n} \alpha q\right]=0} & \text { on } I,\end{cases}
$$

and if $\delta=0$ :

$$
\begin{cases}\Delta q_{C}=0 & \text { in } \Gamma_{C} \\ \Delta q_{E}=0 & \text { in } \Gamma_{E}, \\ q_{C}=0, \quad\left[\gamma \partial_{n} q\right]=0 & \text { on } I .\end{cases}
$$

Both problems (5.4) and (5.5) are scalar elliptic transmission problems. Besides the trivial zero solution, their solutions are the singularities of these problems.

For $\boldsymbol{\psi}$, we obtain from $(5.1),(5.2)(i)$ and $(5.3)(i)$ :

$$
\begin{cases}\operatorname{curl} \boldsymbol{\psi}_{C}=\nabla q_{C}, \operatorname{div}\left(\mu_{C} \boldsymbol{\psi}_{C}\right)=0 & \text { in } \Gamma_{C}, \\ \operatorname{curl} \boldsymbol{\psi}_{E}=\nabla q_{E}, \operatorname{div}\left(\mu_{E} \boldsymbol{\psi}_{E}\right)=0 & \text { in } \Gamma_{E}, \\ {[\boldsymbol{\psi} \times \mathbf{n}]=0, \quad[\mu \boldsymbol{\psi} \cdot \mathbf{n}]=0} & \text { on } I .\end{cases}
$$


Finally for $\mathbf{s}$ we have:

$$
\begin{cases}\operatorname{curl} \mathbf{s}_{C}=\mu_{C} \boldsymbol{\psi}_{C}, \operatorname{div} \mathbf{s}_{C}=q_{C} & \text { in } \Gamma_{C}, \\ \operatorname{curl}_{E} \mathbf{s}_{E}=\mu_{E} \boldsymbol{\psi}_{E}, \operatorname{div} \mathbf{s}_{E}=q_{E} & \text { in } \Gamma_{E}, \\ {[\mathbf{s} \times \mathbf{n}]=0,[\alpha \mathbf{s} \cdot \mathbf{n}]=0} & \text { on } I,\end{cases}
$$

the last transmission condition becoming $\mathbf{s}_{C} \cdot \mathbf{n}=0$ on $I$ if $\delta=0$.

\subsection{The three types of Maxwell singularities}

The Maxwell or eddy current singularities of Type 1 are the solutions of (5.7) with $\boldsymbol{\psi}=0$ and $q=0$. We can see that they are the gradient of scalar singularities: $\mathbf{s}_{C}=\nabla \Phi_{C}$ and $\mathbf{s}_{E}=\nabla \Phi_{E}$ with

$$
\begin{cases}\Delta \Phi_{C}=0 & \text { in } \Gamma_{C}, \\ \Delta \Phi_{E}=0 & \text { in } \Gamma_{E}, \\ {[\Phi]=0, \quad\left[\alpha \partial_{n} \Phi\right]=0} & \text { on } I,\end{cases}
$$

the last transmission condition becoming $\partial_{n} \Phi_{C}=0$ on $I$ if $\delta=0$.

The Maxwell or eddy current singularities of Type 2 are particular solutions of (5.7) with $\boldsymbol{\psi}$ solution of (5.6) and $q=0$. We find that $\boldsymbol{\psi}$ is a gradient: $\boldsymbol{\psi}_{C}=\nabla \Psi_{C}$ and $\boldsymbol{\psi}_{E}=\nabla \Psi_{E}$ with

$$
\begin{cases}\Delta \Psi_{C}=0 & \text { in } \Gamma_{C}, \\ \Delta \Psi_{E}=0 & \text { in } \Gamma_{E}, \\ {[\Psi]=0, \quad\left[\mu \partial_{n} \Psi\right]=0} & \text { on } I .\end{cases}
$$

The solutions of the above transmission problem with real coefficients are linear combinations of terms of the form $\Psi=\rho^{\lambda} \psi(\vartheta)$. Then a particular solution $\mathbf{s}$ associated with such a $\Psi$ is given by (for $\lambda \neq-1$ )

$$
\mathbf{s}=(\lambda+1)^{-1}(\mu \nabla \Psi \times \mathbf{x}-\nabla r)
$$

with $r$ solution of

$$
\begin{cases}\Delta r_{C}=0 & \text { in } \Gamma_{C}, \\ \Delta r_{E}=0 & \text { in } \Gamma_{E}, \\ {[r]=0, \quad\left[\alpha \partial_{n} r\right]=[\alpha \mu](\nabla \Psi \times \mathbf{x}) \cdot \mathbf{n}} & \text { on } I,\end{cases}
$$

the last condition being replaced with $\partial_{n} r_{C}=\mu_{C}\left(\nabla \Psi_{C} \times \mathbf{x}\right) \cdot \mathbf{n}$ on $I$ when $\delta=0$.

The singularities of Type 3 are particular solutions of (5.7), with $\boldsymbol{\psi}$ particular solution of (5.6) and $q$ general solution of (5.5).

In our case, the singularities of Type 3 can be discarded because their divergences form the asymptotic part of the divergence of the solution. Since our solutions are divergence-free, these singularities do not appear in the asymptotics (4.1).

For any singular exponent $\nu^{0} \in \mathfrak{S}^{0}$ of the eddy current problem which is not at the same time an exponent of type 1 and an exponent of type 2 , we have as $\delta \rightarrow 0$ a stable cluster of singular exponents $\nu_{i}^{\delta}$ of the same type tending to $\nu^{0}$.

Let us develop that point for singularities of type 1 (which are the most important ones, since they are the only possible non- $H^{1}$ singularities). Let $\nu^{0} \in \mathfrak{S}^{0}$ 
be of type 1, and let $\gamma$ be a simple contour isolating $\nu^{0}$ from the other elements of $\mathfrak{S}^{0}$. There exists $\delta_{0}>0$ such that for all $\delta \in\left[0, \delta_{0}\right], \gamma \cap \mathfrak{S}^{\delta}=\emptyset$.

Let $\lambda \mapsto \mathfrak{M}^{\delta}(\lambda)$ be the Mellin symbol of problem (5.8) and let $\mathbf{T}_{\gamma+1}^{\delta}$ be the singularity space for potentials $\Phi: \mathbf{T}_{\gamma+1}^{\delta}$ is spanned by the residues

$$
\frac{1}{2 i \pi} \int_{\gamma+1} \rho^{\lambda} \mathfrak{M}^{\delta}(\lambda)^{-1} \mathbf{G}(\lambda) \mathrm{d} \lambda
$$

for all holomorphic $\lambda \mapsto \mathbf{G}(\lambda)$. Then $\mathbf{S}_{\gamma}^{\delta}=\nabla \mathbf{T}_{\gamma+1}^{\delta}$.

\section{References}

[1] H. Ammari, A. Buffa and J.-C. Nédélec, A justification of eddy currents model for the Maxwell equations, SIAM J. Appl. Math. 60 (2000), 1805-1823.

[2] A. Bossavit, Two dual formulations of the 3D eddy-current problem, COMPEL 4 (1985), 103-116.

[3] A. Bossavit, Electromagnétisme en vue de la modélisation, Springer Verlag, 1993.

[4] M. Costabel and M. Dauge, Singularités d'arêtes pour les problèmes aux limites elliptiques. In Journées "Équations aux Dérivées Partielles" (Saint-Jean-de-Monts, 1992). École Polytech., Palaiseau, 1992, Exp. No. IV, 12p.

[5] M. Costabel and M. Dauge, Singularities of Maxwell's equations on polyhedral domains, Arch. Rational Mech. Anal. 151 (2000), 221-276.

[6] M. Costabel, M. Dauge and S. Nicaise, Singularities of Maxwell interface problems, RAIRO Modél. Math. Anal. Numér. 33 (1999), 627-649.

[7] M. Costabel, M. Dauge and S. Nicaise, Singularities of eddy current problems, Preprint of the Newton Institute 03019, Cambridge (2003).

[8] I. Gohberg and E. Sigal, An operator generalization of the logarithmic residue theorem and the theorem of Rouché, Math. USSR Sbornik 13 (4) (1971), 603-625.

[9] R. Hiptmair, Symmetric coupling for eddy currents problems, SIAM J. Numer. Anal. 40 (2002), 41-65.

[10] T. Kato, Perturbation theory for nullity, deficiency and other quantities of linear operators, J. Analyse Math. 6 (1958), 261-322.

[11] V. A. Kondrat'ev, Boundary-value problems for elliptic equations in domains with conical or angular points, Trans. Moscow Math. Soc. 16 (1967), 227-313.

[12] J.-L. Lions and E. Magenes, Problèmes aux limites non homogènes et applications, Vol. 1. Dunod, Paris, 1968.

[13] V. G. Maz'ya, B. A. Plamenevskii, On the coefficients in the asymptotic of solutions of the elliptic boundary problem in domains with conical points, Amer. Math. Soc. Trans. (2) 123 (1984), 57-88.

[14] V. G. Maz'ya and J. Rossmann, On a problem of Babuška (Stable asymptotics of the solution to the Dirichlet problem for elliptic equations of second order in domains with angular points), Math. Nachr. 155 (1992), 199-220.

[15] S. Nicaise, Polygonal interface problems, Peter Lang, Berlin, 1993. 
[16] B. Schmutzler, Branching asymptotics for elliptic boundary value problems in a wedge, in "Boundary value problems and integral equations in nonsmooth domains (Luminy, 1993)", M. Costabel, M. Dauge, and S. Nicaise, eds., Dekker, New York, (1995), 255-267.

IRMAR, Université de Rennes 1, Campus de Beaulieu, 35042 Rennes, France

E-mail address: costabel@univ-rennes1.fr

IRMAR, Université de Rennes 1, Campus de Beaulieu, 35042 Rennes, France

E-mail address: dauge@univ-rennes1.fr

Université de Valenciennes et du Hainaut Cambrésis, MACS, Le Mont Houy, 59313

Valenciennes Cedex 9, France

E-mail address: snicaise@univ-valenciennes.fr 